\title{
Multi-step pretreatment as an eco-efficient pretreatment method for the production of cellulose nanofiber from oil palm empty fruit bunch
}

\author{
Liana Noor Megashaha, Hidayah Ariffin, ${ }^{a, b^{*}}$, Mohd Rafein Zakaria ${ }^{a}$, Mohd Ali Hassan ${ }^{\mathrm{a}}$ \\ ${ }^{a}$ Department of Bioprocess Technology, Faculty of Biotechnology and Biomolecular Sciences, Universiti Putra Malaysia, 43400 \\ UPM Serdang, Selangor, Malaysia \\ ${ }^{b}$ Laboratory of Biopolymer and Derivatives, Institute of Tropical Forestry and Forest Products (INTROP), Universiti Putra \\ Malaysia, 43400 UPM Serdang, Selangor, Malaysia
}

Received 3rd August 2018 / Accepted 29th August 2018

\begin{abstract}
Cellulose nanofiber (CNF) characteristics could be influenced by the pretreatment process during cellulose isolation, and generally pretreatment is conducted using harsh, less eco-efficiency chemical pretreatment. In this study, multi-step pretreatment method was evaluated for its eco-efficiency and compared with the conventional soda pulping method for cellulose isolation from oil palm empty fruit bunch (OPEFB). CNF developed from the celluloses pretreated by these methods were characterized. Some amount of hemicellulose residue left after the pretreatments whereby multi-step method showed higher amount of hemicellulose residue. This affected the diameter size of CNF obtained in which CNF from multi-step pretreated cellulose had smaller diameter range $(13-33 \mathrm{~nm})$ compared to that of soda pulping (18-52 $\mathrm{nm})$. Crystallinity, thermal stability and degree of polymerization of the two CNFs are comparable. The superior characteristics of CNF obtained from multi-step pretreatment method, in addition to its eco-efficiency characteristic as evaluated based on the two key elements of eco-efficiency, namely, process re-engineering and by-products valorization, have recommended multi-step pretreatment method as a promising method for cellulose isolation from lignocellulose.
\end{abstract}

Keywords: eco-efficient, cellulose isolation, multi-step pretreatment, soda pulping, oil palm empty fruit bunch, cellulose nanofiber

\section{INTRODUCTION}

One approach in Environmental Biotechnology is to introduce a process which makes optimal use of natural resources by biomass recycling and utilization, as well as waste minimization, with the aim to avoid environmental degradation towards sustainable development. Waste to wealth idea through utilization of agricultural residues for value-added products has been one of the main topics of interest. In sustainable development, eco-efficiency is important as the concept highlights the environmental improvement in practices and processes without compromising the economic benefits (WBCSD, 2006). Nanocellulose is one of the materials which could be produced from agricultural residues. It is a cellulose-based material with one of its dimension in nanosize. Cellulose in nature is a polysaccharide consisting anhydroglucose $\left(\mathrm{C}_{6} \mathrm{H}_{10} \mathrm{O}_{5}\right)$ repeating units which are linked together by $\beta$-glucosidic bond (Krässig et al., 2000). Nanocellulose has

* Author for correspondence: Dr. Hidayah Ariffin, Department of Bioprocess Technology, Faculty of Biotechnology and Biomolecular Sciences, Universiti Putra Malaysia, 43400 UPM Serdang, Selangor, Malaysia. Email-hidayah@upm.edu.my 
various potential applications, namely as reinforcement material in biocomposite, for biomedical applications, food rheology control and paper packaging products (Chang et al., 2012; Szczesna-Antczak et al., 2012; Iwamoto et al., 2014). Nanocellulose from plant resources can be classified into cellulose nanocrystals (CNC) and cellulose nanofibrils (CNF) (Dufresne, 2013).

Cellulose isolation is an important procedure prior to nanocellulose production since most of the agricultural residues are in lignocellulosic form, which consists not only of cellulose but also of lignin and hemicellulose. These two components may interfere the fibrillation and hydrolysis process which could cause failure in $\mathrm{CNC}$ and $\mathrm{CNF}$ production. Common industrial process for cellulose isolation is through pulping process. For example, in soda pulping, the use of highly concentrated alkali has caused problems in wastewater discharge. Moreover, high concentration of chemicals used may result in corrosion of the digester, causing long standing environmental problems (Sharma et al., 2012). Pulping process also requires high energy as the process involves the use of high temperature and pressure.

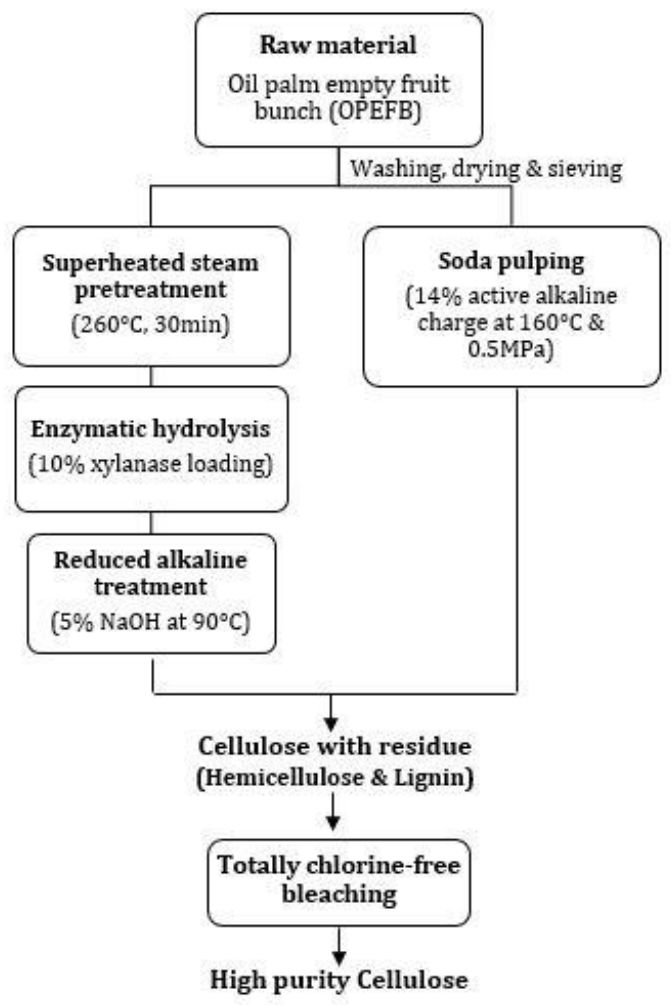

Figure 1. Cellulose isolation from oil palm empty fruit bunch by two different methods.
In tropical countries like Malaysia, palm oil industry is one of the biggest contributors in gross national income (GNI). Due to active production of palm oil, abundance of oil palm biomass is being generated at the mill and at the plantation. Oil palm empty fruit bunch (OPEFB), one of the solid biomass generated at the mill has potential to be the feedstock for nanocellulose production due to its high cellulose content (Fatah et al., 2014). Eco-efficient utilization of OPEFB which is considered as waste at the mill for nanocellulose production could contribute to sustainable development.

This paper aims to introduce an eco-efficient process for the production of nanocellulose (CNF) from OPEFB by evaluating two methods for isolating cellulose from the OPEFB (Figure 1), and the effect of the methods on the characteristics of the nanocellulose produced.

\section{MATERIALS AND METHODS}

Materials. Oil palm empty fruit bunches (OPEFB) were supplied by Seri Ulu Langat Palm Oil Mill, Dengkil Selangor, Malaysia. The obtained samples were thoroughly washed and dried. The dried fibers were cut to a length of about $30-50 \mathrm{~mm}$ and stored prior to pretreatment. Untreated OPEFB fibers contained 37.1\% cellulose, $39.9 \%$ hemicellulose and 18.6\% lignin as determined according to TAPPI standard analysis. Sodium hydroxide was purchased from QRec Chemical, Malaysia while hydrogen peroxide and acetic acid were purchased by J.T. Baker on industrial grade chemicals. Meanwhile, the sulfuric acid (95-98\%) was by Sigma Aldrich. The commercial xylanase (Pulpzyme ${ }^{\circledR}$ HC 2500) was supplied by Novozymes A/S Denmark with unit activity of $2500 \mathrm{AXU} / \mathrm{g}$.

Pretreatment of oil palm biomass. In comparative studies, two types of pretreatment methods namely multi-step pretreatment and soda pulping (conventional pretreatment method) were carried out. For bleaching, totally chlorine free (TCF) chemical which is known as peracetic acid was used. Peracetic acid solution was prepared by mixing hydrogen peroxide and acetic acid with addition of sulfuric acid as catalyst. The 
chemical preparation is as being described in previous work (Megashah et al., 2018).

\section{Multi-step pretreatment. Multi-step} pretreatment is a combined pretreatment method consisted of superheated steam (SHS) treatment, enzymatic hydrolysis and reduced concentration alkaline treatment. SHS pretreatment was conducted using a lab scale superheated steam oven (QF-5200C, Naomoto Corporation, Osaka, Japan) and with the aim to partially remove the hemicellulose (Nordin et al., 2013). About $30 \mathrm{~g}$ of fibers were placed on stainless steel mesh; the temperature and retention time within the oven chamber were set at $260^{\circ} \mathrm{C}$ and $30 \mathrm{~min}$, respectively. The pretreatment was conducted at atmospheric pressure. Subsequently, enzymatic pretreatment was conducted using xylanase at 2 $\mathrm{wt} \%$ of SHS-treated OPEFB in sodium phosphate buffer ( $\mathrm{pH} 5.5)$, using 10\% (v/v) of enzyme loading. The hydrolysis was conducted at $55^{\circ} \mathrm{C}$ and $160 \mathrm{rpm}$ for 24 hours. Afterwards, alkaline pretreatment was conducted using $5 \%$ $(\mathrm{w} / \mathrm{v})$ of sodium hydroxide. The ratio of solid to liquid was fixed at 1:10 (w:w). The pretreatment was done at $90^{\circ} \mathrm{C}$ for 1 hour. Solid residues obtained were then filtered and washed several times with tap water until the $\mathrm{pH}$ became neutral. The fiber obtained was dried at $60^{\circ} \mathrm{C}$ for overnight prior to chemical analysis and TCF bleaching.

Soda pulping. Soda pulping was conducted as reported earlier (Megashah et al., 2018). Fiber samples were mixed with alkaline solution $(14 \%$ $\mathrm{NaOH})$ at ratio of $1: 4$ and the pulping process took place at $160^{\circ} \mathrm{C}$ with pressure at about 0.5 $\mathrm{MPa}$ in a 15L lab scale twin digester (model GTD15L, GIST Co Ltd., Korea) for one hour. After cooling, the cellulose pulp were washed several times using tap water until the $\mathrm{pH}$ became neutral. The pulp underwent spinning to remove water and dried at $60^{\circ} \mathrm{C}$ overnight. The treated samples were then subjected to chemical analysis and TCF bleaching.

TCF bleaching. Pretreated fibers from multistep and soda pulping pretreatment methods were subjected to bleaching step using TCF chemicals. Bleaching was carried out at $70^{\circ} \mathrm{C}$ for overnight. After bleaching, the solid residues (cellulose) were collected by washing it several times until neutral $\mathrm{pH}$ was achieved.

\begin{abstract}
Nanofibrillation of OPEFB-cellulose by wet disc mill. Cellulose nanofiber (CNF) was produced by using a high shear ultrafine friction grinder, or wet disc mill, WDM (Multi mill, Grow Engineering, Adachi-ku, Tokyo, Japan). OPEFBcellulose was first suspended in water for 72 hours before fibrillation using the WDM at $1 \mathrm{wt} \%$ solid content. The suspension was passed through the grinder in between the static and rotating stones for 10 cycles. The clearance between the stones were adjust in between 30 to 50 micron, and rotating stone speed was set at $1,800 \mathrm{rpm}$. The obtained nanocellulose slurry was then stored in a cold room at temperature of $4^{\circ} \mathrm{C}$ prior to characterization.
\end{abstract}

Chemical compositional analysis. Chemical compositional analysis was conduct based on Technical of Pulp \& Paper Industry (TAPPI) Standard Test Method. Composition included determination of lignin content (T222 om-88), cellulose and hemicellulose content (TAPPI useful method 249-75).

Thermal stability. Thermal stability of the samples was analyzed using a thermogravimetric analyzer (TGA) model EXSTAR6000 (Hitachi High-Tech Science Corporation, Tokyo, Japan). The heating was set at temperature range in between $50^{\circ} \mathrm{C}$ to $550^{\circ} \mathrm{C}$ with heating rate of $10^{\circ} \mathrm{C} / \mathrm{min}$. Pure nitrogen as the inert gas was purged at flow rate of $100 \mathrm{ml} / \mathrm{min}$ during the analysis.

Morphological analysis. Morphology of OPEFB-CNF was observed using a fieldemission scanning electron microscopy (JEOL 7500 FESEM). The samples were coated with platinum using a vacuum sputter coater prior to FE-SEM viewing.

$\boldsymbol{X}$-ray diffraction analysis. Crystallinity of samples was measured using an X-ray Diffractometer (model Rigaku MiniFlex 600) at $40 \mathrm{kV}-15 \mathrm{~mA}$. The reflection mode was conducted by taking $2 \theta$ scan range in between $5^{\circ}$ to $50^{\circ}$ at 
$2.00^{\circ} / \mathrm{min}$. Crystallinity index (CrI) was calculated (Yasim-Anuar et al., 2017) as follows:

$$
\mathrm{CrI}=\frac{\mathrm{I}_{002}-\mathrm{I}_{\mathrm{am}}}{\mathrm{I}_{002}} \times 100
$$

where, $\mathrm{I}_{002}$ is taking from the diffraction intensity at $2 \theta=22^{\circ}$ and $I_{a m}$ at the intensity of $2 \theta=18^{\circ}$.

Degree of polymerization. Degree of polymerization (DP) of cellulose samples was measured using a viscometer (TAPPI T230, ISO 5351). The OPEFB-cellulose was diluted in copper (II) ethylenediamine (CED) solution prepared with a ratio of 0.01:1:1 (OPEFBcellulose:distilled water:CED). The solution was mixed and stirred until all fibers were completely dissolved. The OPEFB-cellulose solution was then poured into an Ubbelohde viscometer tube (Type 231) and the measurement was carried out at $25^{\circ} \mathrm{C}$. The molecular mass of cellulose samples was determined using Mark-Houwink equation as shown below:

$$
[\eta]=\mathrm{KM}^{\alpha}
$$

where $[\eta]$ is the intrinsic viscosity and $\mathrm{M}$ is the molecular mass. $K=0.42$ and $\alpha=1$ are constant values of CED solvent (Yasim-Anuar et al., 2017)

\section{RESULTS AND DISCUSSION}

\section{Effect of pretreatments on chemical} composition of pretreated fiber. Table 1 exhibits cellulose, hemicellulose and lignin composition in the fiber after pretreatments. TCF pretreatment was conducted for bleaching purpose, aimed at lignin removal. The attributed cellulose represents $\alpha$-cellulose composition after separation of lignin and hemicellulose. After cellulose isolation process, the cellulose composition in the fiber may reflect the purity of cellulose, as non-cellulosic components can be regarded as impurities.

Untreated OPEFB composed of about 37\% of cellulose. After pretreatments for hemicellulose removal (multi-step and soda pulping without TCF pretreatment), the cellulose purity increased to $68.6 \%$ and $78.3 \%$, respectively, for multi-step and soda pulping methods. Higher lignin content was present after multi-step pretreatment compared to soda pulping, attributed to lignin aromatic structure which is removed at severe alkaline conditions (high concentration, high temperature and longer reaction time) (Zeng et al., 2014). Combination of multi-step pretreatment with TCF pretreatment yielded OPEFB with cellulose purity of $83.7 \%$, while combined soda pulping and TCF pretreatment gave $93 \%$ purity. Lignin was almost completely removed in both samples. Despite of almost $10 \%$ difference in cellulose purity, this difference is considered small considering the big difference in reaction conditions. Reduced alkali concentration and atmospheric pressure were used in multi-step pretreatment, suggesting that severe alkaline conditions could actually be omitted during pretreatment. Nevertheless, effect of residual lignin and hemicellulose on the formation of $\mathrm{CNF}$ will be discussed in the next section.

\begin{tabular}{|c|c|c|c|c|c|}
\hline $\begin{array}{l}\text { Sample } \\
\text { ID }\end{array}$ & Pretreatment & Cellulose (\%) & $\begin{array}{c}\text { Hemicellulose } \\
(\%)\end{array}$ & $\begin{array}{c}\text { Lignin } \\
(\%)\end{array}$ & References \\
\hline Sample 1 & Untreated OPEFB & $37.1 \pm 4.4$ & $39.9 \pm 0.8$ & $18.6 \pm 1.3$ & $\begin{array}{l}\text { Megashah } e t \\
\text { al., } 2018\end{array}$ \\
\hline Sample 2 & $\begin{array}{c}\text { Multi-step } \\
\text { (without TCF) }\end{array}$ & $68.6 \pm 0.5$ & $15.6 \pm 2.9$ & $16.9 \pm 0.6$ & This study \\
\hline Sample 3 & $\begin{array}{l}\text { Soda pulping } \\
\text { (without TCF) }\end{array}$ & $78.3 \pm 1.4$ & $17.6 \pm 0.3$ & $4.1 \pm 1.4$ & This study \\
\hline Sample 4 & $\begin{array}{l}\text { Multi-step } \\
\text { (with TCF) }\end{array}$ & $83.7 \pm 2.5$ & $14.5 \pm 1.1$ & $0.7 \pm 0.4$ & This study \\
\hline Sample 5 & $\begin{array}{l}\text { Soda pulping } \\
\text { (with TCF) }\end{array}$ & $93.0 \pm 0.5$ & $7.0 \pm 0.5$ & $0.0 \pm 0.0$ & $\begin{array}{l}\text { Megashah et } \\
\text { al., } 2018\end{array}$ \\
\hline
\end{tabular}

Table 1. Chemical composition of untreated and pretreated OPEFB.

Data on composition (\%) are representative of three replicates. 


\section{Effect of residual lignin and hemicellulose on} $\boldsymbol{C N F}$ formation. In the present work, obtained cellulose was mechanically nanofibrillated using a wet disc mill to produce CNF. Only $1 \mathrm{wt} \%$ of cellulose solid was used during the processing since nanofibrillation involves the breakage of hydrogen bonds in between cellulose microfibrils which eventually caused the nanocellulose to have very high water holding capacity, and hence a thick, gel-like nanocellulose suspension in water will be produced. Figure 2 shows the FE-SEM micrographs of $\mathrm{CNF}$ produced from pretreated OPEFB cellulose. Untreated OPEFB and pretreated OPEFB obtained after multi-step pretreatment without TFC (samples 1 and 2, respectively) failed to be nanofibrillated, due to high lignin content in the samples. Lignin acts as glue which causes the cellulose and hemicellulose to be strongly bound (Abdel-Halim, 2014), and hence it is difficult to obtain nanofiber from these samples. Effect of lignin composition can be seen clearly in samples 3, 4 and 5. For samples 4 and 5 which had undergone lignin removal by TCF pretreatment, the CNF produced had width size of less than $50 \mathrm{~nm}$ (Figures $2 \mathrm{a}$ and $2 \mathrm{~b}$ ). On the other hand, sample 3 which was prepared by soda pulping without TCF pretreatment had larger width size range, with some fibers obtained had width size of more than $100 \mathrm{~nm}$ (Figure 2c). This could be explained by the presence of some amount of lignin in sample 3, which prevented further nanofibrillation to occur.

Meanwhile, effect of hemicellulose on nanofibrillation can be seen by comparing samples 4 and 5 (Figures $2 \mathrm{a}$ and $2 \mathrm{~b}$ ). Sample 4 which was prepared by multi-step pretreatment had smaller width size (13-33 nm based on histogram), as compared to sample 5 (18-52 nm). Similar observation has been reported earlier, in which hemicellulose was found to assist in mechanical nanofibrillation. This can be explained by the avoidance of hornification effect, in which the residual hemicellulose would adhere to the nanofiber and avoid agglomeration (Iwamoto et al., 2008; Norrrahim et al., 2018).
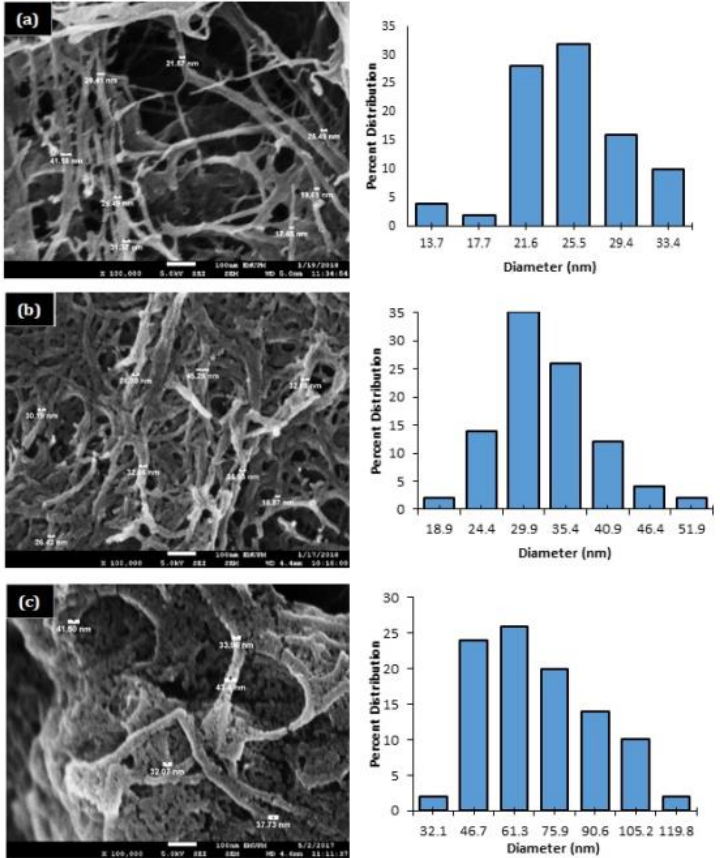

Figure 2. FE-SEM images of CNF-OPEFB at 100,000X magnification. (a) CNF from multi-step with TCF (sample 4); (b) CNF from soda pulping. With TCF (sample 5); (c) CNF from soda pulping without TCF (sample 3).

Characterization of cellulose nanofiber produced. Table 2 shows the characteristics of CNF produced from multi-step and soda pulping pretreatment methods. CNF from multi-step pretreated cellulose showed a slightly lower thermal stability compared to CNF from soda pulp, as shown by Tmax value which was obtained from differential thermogravimetry (DTG) thermograms. This observation could be related to the higher hemicellulose content in multi-step pretreated cellulose, since it is known that hemicellulose is a less thermally stable component compared to cellulose. In terms of crystallinity, CNF from soda pulp had higher crystallinity $(82 \%)$, but when it comes to application of the $\mathrm{CNF}$, this crystallinity value could give comparable effect to that of multi-step pretreated CNF which had crystallinity of $70 \%$. It has been reported that crystallinity of nanocellulose at $70 \%$ and above is considered high (Isogai et al., 2011) and will be advantageous in improving the mechanical properties of material. In term of DP, it is interesting to note that the DP of cellulose from the two pretreatment methods were slightly different. Lower DP value for soda pulp CNF may indicate 
that some cellulose was degraded during soda pulping process, compared to the multi-step pretreated CNF. This is expected since soda pulping involved the use of highly concentrated alkali and harsh conditions (high temperature and pressure). Substantially, reduction in DP may result in the formation of shorter nanofibrils, lowering the aspect ratio and hence could negatively affect the performance of $\mathrm{CNF}$ as reinforcement material (Qin et al, 2016).

Table 2. CNF properties of OPEFB-treated from multi-step and soda pulping pretreatment.

\section{Multi-step (with TCF) Soda pulping (with TCF)}

$\begin{array}{ccc}\begin{array}{c}\text { Maximum thermal degradation } \\ \text { temperature, } \mathrm{T}_{\max }\left({ }^{\circ} \mathrm{C}\right)\end{array} & 350 & 360 \\ \text { Crystallinity index }(\%) & 70.0 & 82.0 \\ \text { Degree of polymerization (DP) } & 1,410 & 1,300\end{array}$

Evaluation of pretreatment methods. Both of the cellulose isolation methods were evaluated in term of its eco-efficiency for the production of $\mathrm{CNF}$ from OPEFB. Among the key elements of eco-efficiency are to re-engineer the process and revalorize by-products (WBCSD, 2006). To further elaborate this, five related aspects were evaluated: total unit operations involved, pretreatment conditions, energy requirement, waste production and management, and potential for by-products valorization. The first three aspects relate to process re-engineering, in which the conventional soda pulping process which is commonly used in cellulose isolation can be reengineered in order to meet eco-efficiency concept. By-products revalorization is another important element, which is discussed through the two last aspects.

In term of total unit operations involved during pretreatment, soda pulping involves only one unit operation which is digester for pulping. Multi-step pretreatment requires three steps which are superheated steam pretreatment, enzymatic pretreatment and low concentration alkaline pretreatment. It seems like soda pulping is a simpler pretreatment methods as it does not involve multiple stages of pretreatment. Nevertheless, pretreatment conditions in soda pulping are harsh, in which the process involves the use of high pressure, high temperature and high alkaline strength. It is less eco-friendly compared to the multi-step pretreatment, as this process will require higher energy, as more energy will be needed to provide high pressure system during the pretreatment. SHS, despite of its steam pretreatment, is a process conducted at atmospheric pressure. Therefore it is safer and requires less energy compared to high pressure condition in digester. Nevertheless, due to the involvement of three unit operations for multistep pretreatment, the method is also lacking in term of energy requirement, similar to soda pulping. However, for CNF production from oil palm biomass, energy requirement may not be an issue since the palm oil mills are currently generating excess energy in the form of high pressure steam (Abdullah et al., 2016). It is hence necessary to locate the $\mathrm{CNF}$ processing facilities nearby the palm oil mill so that this excess steam can be tapped and utilized for the process.

Table 3. Impact of the pretreatment method on the following aspects.

\begin{tabular}{|c|c|c|}
\hline Aspect & Multi-step & $\begin{array}{c}\text { Soda } \\
\text { pulping }\end{array}$ \\
\hline $\begin{array}{c}\text { Total unit } \\
\text { operations }\end{array}$ & $\mathbf{x}$ & $\checkmark$ \\
\hline $\begin{array}{c}\text { Pretreatment } \\
\text { conditions }\end{array}$ & $\checkmark$ & $\mathbf{x}$ \\
\hline $\begin{array}{c}\text { Energy } \\
\text { requirement }\end{array}$ & $\mathbf{x}$ \\
\hline $\begin{array}{c}\text { Waste production } \\
\text { and management }\end{array}$ & $\checkmark$ & $\mathbf{x}$ \\
\hline $\begin{array}{c}\text { By-products } \\
\text { valorization } \\
\text { potential }\end{array}$ & $\checkmark$ & $\mathbf{x}$ \\
\hline
\end{tabular}

$\checkmark$ is good and $\mathbf{x}$ is poor. 
In terms of waste production and management, having a three-unit operation could be an advantage for multi-step pretreatment, since the by-products from each step will not be mixed and this provides better waste management. In soda pulping, all degraded products from hemicellulose, lignin and cellulose will be mixed together as black liquor, which causes difficulty for waste treatment or by-product utilization. Black liquor has been an issue for many pulp industries, as it could give an adverse effect and is toxic to the ecology. The pulp mill owners face a lot of difficulties as they need to pass very stringent environmental standard for wastewater discharge (Ashrafi et al., 2015). Additionally, byproducts valorization potential for this process is difficult due to the nature of the black liquor. This is the reason soda pulping method does not obtain any point for these two aspects (Table 3). Meanwhile, multi-step pretreatment allows easier separation of by-products. For instance, it has been reported that the by-products from SHS pretreatment could be used as antimicrobial agent as the by-product can be collected as distillate during the SHS pretreatment (Sharip et al., 2016). Enzymatic pretreatment may produce degraded hemicellulose portion which may appear as oligosaccharides or sugars, which have several potential applications such as fermentation substrate (Ahmad et al., 2018; Lachke, 2002).

\section{CONCLUSION}

Eco-efficiency concept in nanocellulose production from oil palm biomass can be practiced by re-engineering the conventional cellulose isolation process. This could be done by considering the effects of the re-engineered process on the environment, without compromising the quality of the nanocellulose produced. Herewith, the conventional cellulose isolation methods via soda pulping was revised, and compared with a multi-step pretreatment method which is more eco-friendly and less harsh. It was shown that the characteristics of the cellulose nanofiber produced by multi-step pretreated cellulose were excellent, almost similar to that produced from soda pulp.

\section{ACKNOWLEDGEMENTS}

This work was financially supported by Universiti Putra Malaysia (UPM) through Geran Putra-IPS (9519300) and Putra Berimpak (9523000). The authors would like to thank Seri Ulu Langat Palm Oil Mill for the provision of oil palm empty fruit bunch (OPEFB) for this study.

\section{REFERENCES}

Abdel-Halim, E. S. 2014. Chemical modification of cellulose extracted from sugarcane bagasse: Preparation of hydroxyethyl cellulose. Arabian Journal of Chemistry 7(3): 362371.

Ahmad, N., Zakaria, M. R., Mohd Yusoff, M. Z., Fujimoto, S., Inoue, H., Ariffin, H., Shirai, Y. 2018. Subcritical watercarbon dioxide pretreatment of oil palm mesocarp fiber for xylooligosaccharide and glucose production. Molecules (Basel, Switzerland) 23(6).

Ashrafi, O., Yerushalmi, L., \& Haghighat, F. 2015. Wastewater treatment in the pulp-and-paper industry: a review of treatment processes and the associated greenhouse gas emission. Journal of Environmental Management 158: 146-157.

Chang, F., Lee, S. H., Toba, K., Nagatani, A., \& Endo, T. 2012. Bamboo nanofiber preparation by $\mathrm{HCW}$ and grinding treatment and its application for nanocomposite. Wood Science and Technology 46(1-3): 393-403.

Dufresne, A. 2013. Nanocellulose : a new ageless bionanomaterial. Materials Today 16(6): 220-227.

Fatah, I. Y. A., Abdul Khalil, H. P. S., Hossain, M. S., Aziz, A. A., Davoudpour, Y., Dungani, R., \& Bhat, A. 2014. Exploration of a chemo-mechanical technique for the isolation of nanofibrillated cellulosic fiber from oil palm empty fruit bunch as a reinforcing agent in composites materials. Polymers 6(10): 2611-2624.

Isogai, A., Saito, T., \& Fukuzumi, H. 2011. TEMPO-oxidized cellulose nanofibers. Nanoscale 3(1): 71-85.

Iwamoto, S., Abe, K., \& Yano, H. 2008. The effect of hemicelluloses on wood pulp nanofibrillation and nanofiber network characteristics. Biomacromolecules 9: 1022-1026.

Iwamoto, S., Yamamoto, S., Lee, S. H., Ito, H., \& Endo, T. 2014. Mechanical and thermal properties of polypropylene composites reinforced with lignocellulose nanofibers dried in melted ethylene-butene copolymer. Materials 7(10): 69196929.

Krässig, H., Schurz, J., Steadman, R. G., Schliefer, K., \& Albrecht, W. 2000. Cellulose. In Ullmann's Encyclopedia of Industrial Chemistry. American Cancer Society.

Lachke, A. 2002. Biofuel from D-xylose - the second most abundant sugar. Resonance 7(5): 50-58.

Megashah, L. N., Ariffin, H., Zakaria, M. R., \& Hassan, M. A. 2018. Properties of cellulose extract from different types of oil palm biomass. IOP Conference Series: Materials Science and Engineering 368: 012049.

Nordin, N. I. A. A., Ariffin, H., Andou, Y., Hassan, M. A., Shirai, Y., Nishida, H., Ibrahim, N. A. 2013. Modification of oil palm mesocarp fiber characteristics using superheated steam treatment. Molecules 18(8): 9132-9146.

Norrrahim, M. N. F., Ariffin, H., Yasim-Anuar, T. A. T., Ghaemi, F., Hassan, M. A., Ibrahim, N. A., Yunus, W. M. Z. W. 
2018. Superheated steam pretreatment of cellulose affects its electrospinnability for microfibrillated cellulose production. Cellulose 25(7): 3853-3859.

Qin, Y., Qiu, X., \& Zhu, J. Y. 2016. Understanding longitudinal wood fiber ultra-structure for producing cellulose nanofibrils using disk milling with diluted acid prehydrolysis. Scientific Reports 6: 35602.

Sharip, N. S., Ariffin, H., Hassan, M. A., Nishida, H., \& Shirai, Y. 2016. Characterization and application of bioactive compounds in oil palm mesocarp fiber superheated steam condensate as an antifungal agent. RSC Advances 6(88): 84672-84683.

Sharma, A., Sulaxna, S. \& Singh, A. K. 2012. Corrosion investigations due to increased sulfidity in digester house of paper mill. Journal of Materials and Environmental Science 3(6): 1009-1018.

Abdullah, S. S. S., Shirai, Y., Ali, A. A. M., Mustapha, M., Hassan M. A. 2016. Case study: Preliminary assessment of integrated palm biomass biorefinery for bioethanol production utilizing non-food sugars from oil palm frond petiole. Energy Conversion and Management 108: 233-242.

Szczesna-Antczak, M., Kazimierczak, J., \& Antczak, T. 2012. Nanotechnology-methods of manufacturing cellulose nanofibres. Fibres and Textiles in Eastern Europe 91(2): 8-12.

WBCSD, Eco-efficiency learning module. World Business Council for Sustainable Development/Five Winds International, 2006. ISBN: 2-940240-84-1.

Yasim-Anuar, T. A. T., Ariffin, H., Norrrahim, M. N. F., \& Hassan, M. A. 2017. Factors Affecting spinnability of oil palm mesocarp fiber cellulose solution for the production of microfiber. Bioresources 12(1): 715-734.

Zeng, Y., Zhao, S., Yang, S., \& Ding, S. 2014. Lignin plays a negative role in the biochemical process for producing lignocellulosic biofuels. Current Opinion in Biotechnology 27: 38-45. 